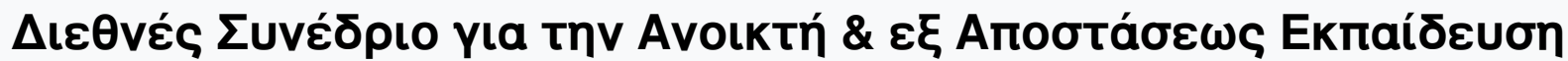

Tón. 5, Ap. 1A (2009)

Open and Distance Education for Global Collaboration \& Educational Development

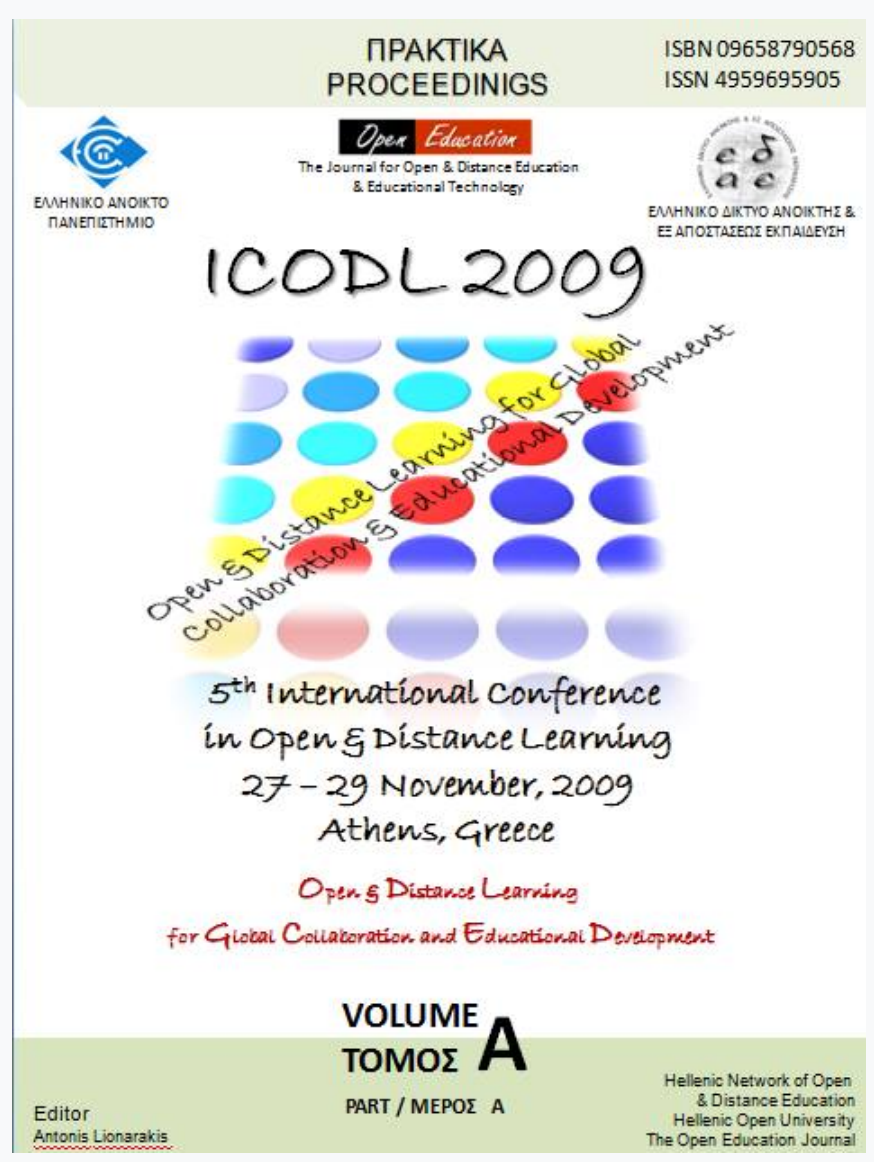

Computer Mediated Discussions in Second Language Writing: Intertextual Connections Iona SARIEVA

doi: $10.12681 /$ icodl. 497 


\title{
Computer Mediated Discussions in Second Language Writing: Intertextual Connections
}

\author{
Iona SARIEVA \\ Ph.D.,Sofia University, Bulgaria \\ US Fulbright scholar, Second Language Acquisition and Instructional Technology \\ iona_sarieva@hotmail.com
}

\begin{abstract}
This quantitative study aims to shed lights on the specifics of computer mediated communication (CMC) within the context of the second language acquisition (SLA) writing processes by examining the pre-writing interactions and the first drafts of 60 English as a Second Language adult learners. These interactions took place either in a synchronous (SCMC) or asynchronous computer-mediated communication (ACMC) environment. The focus was on the lexical intertextual connections evident when the prewriting discussions of the learners were compared with the subsequent drafts accounting for the mode communication. The lexical items of the pre-wring discussion and the first drafts were statistically analyzed by applying a $t$-test. This analysis revealed that there was a statistically significant difference $(\alpha=.05)$ between the ACMC and SCMC groups in terms of pre-writing discussion and first draft lexical intertextual connections. The participants who conducted their pre-writing discussions in an ACMC mode were more likely to use lexical items from the discussion in their first drafts as compared to the SCMC participants. The researcher believes that these findings can inform distance and computer-enhanced education in general as well as language learning and further the research in this area.
\end{abstract}

\section{Introduction}

Although this article addresses topics that are closely related to language acquisition, the findings that it presents might support the better understanding of the communication process in any educational settings when computer interaction is involved within the context of a writing project. In the Second Language Acquisition (SLA) research literature on English as a Second Language (ESL) writing the importance of approaching writing as a process situated into social contexts is viewed as essential for understanding how students engage in writing and acquire writing skills (Grabe \& Kaplan, 1996; Kent, 1999, Matsuda, 2003). Accounting for the social nature of writing allows for the recognition of the significance of communication events that occur among learners and between learners and the instructor, as well as for their impact on text construction. These communication events differ in various discourses such as oral, written, and computer mediated communication discourse (Swaffar, 1998; Warschauer, 1999). It is essential to note that, within the social context, the texts are viewed not as 
isolated entities but rather as parts of a complex community embedded networks; thus, calling for attention to the connections that exist within these networks and the role of these connections in the process of establishing second language writing skills. Further in this introductory section a brief overview of literature related to writing processes in SLA, intertextuality, and collaboration in computer mediated communication (CMC) environments will be presented.

\subsection{CMC Within the Context of Second Language Acquisition}

In the modern society, technology decentralized the workspace, it changed the nature of communication and reshaped the contemporary education. Furthermore, it defined the fast and effective access to and processing of information as a key to success (Leu \& Donald, 2000; Warschauer, 2006). Warschauer (2004) states that these changes are so dynamic in their nature that it is difficult to document and analyze them. He views such an attempt for analysis as crucial for our understanding of how computer-mediated communication can be incorporated into the language teaching process.

Technology transformed the classroom - computer enhanced classes and distance education are becoming more popular. Researchers and practitioners point out that the modern pedagogical practices should aim for the development of literacies that are coherent with these transformations (Kasper, 2000; Warschauer, 1999). Although, it is recognized that today computer-mediated communication (CMC) tools are well known by the younger generation of learners (Miller, 2007), recent research shows that learners are more likely to engage in the recreational use of these tools and a only a small percentage of them are experienced in CMC interactions related to problem-solving professional or academic tasks (Sarieva, 2008; Jin, 2007). Therefore the better understanding of the nature of CMC communication within the context of language learning and writing skills development is needed in order to effectively guide the learning process.

In the contemporary second language research, the positive role of $\mathrm{CMC}$ is recognized; however, in order to support second language educators in the process of implementing CMC into the classroom practices, it is important to consider the specifics of the two modes - synchronous and asynchronous. Researchers found that both modes provide benefits to foreign and second language learners (Beauvois, 1994, 1997; Kern, 1995; Warschauer, 1999). Sotillo (2000) points out that qualitative and quantitative differences between the language outcomes of the asynchronous (ACMC) and synchronous (SCMC) modes exist. Supporting this conclusion are the findings reported by Jepson (2005) about the high interactivity of SCMC interactions as well as by Smith (2003) who concluded that about one-third of the entire SCMC task-based interaction of language learners was devoted to negotiation directly related to the task while the rest of the interaction exchange was directed towards collaborative progression through the communicative task and not directly related to the task topic. In addition, Sarieva (2007) found that the ACMC interaction as compared to the SCMC interaction seem to result in a pre-writing discussion that in form resembles to a higher degree a connected text. These

SECTION A: theoretical papers, original research and scientific articles 
differences should be taken into account when planning to incorporate CMC into the curricula so the CMC activities correspond with the overall goals of the curriculum as well as with the objectives of the specific instruction activities.

\subsection{Writing as a Socially Embedded Process}

In the writing-as-a-process approach literature, writing is understood as a process of text construction in which learners' writing ability and expertise are in constant development (Faigley, 1994; Grabe \& Kaplan, 1996; Reither, 1994). This understanding of writing is furthered by Post-process theory: while "the fundamental observations that an individual produces text by means of a writing process has not been discarded" (Petraglia, 1999, p. 53), writing is viewed as a process which is a socially embedded phenomenon. This process presents a rich context with various elements - writers, genres, audiences, cultural practices, and social interactions (Kent, 1999; Petraglia, 1999; Reither, 1994). Within this context, collaboration is recognized as an essential component of writing instruction that promotes the creation of writing communities within the class (Grabe \& Kaplan, 1996).

The studies discussed in the comprehensive writing research reviews by Ferris (2003), Silva and Matsuda (2001), Ferris and Hedgcock (1998), and Grabe and Kaplan (1996), mainly address the collaboration in the writing process after the preparation for writing and planning are completed, focusing on collaborative writing as well as teacher and peer response. Thus, there is lack of research that would reveal how students move through the continuum of the writing process with consideration of the connections they establish between texts created at the initial points of the process.

\subsection{Intertextuality in SLA}

The importance of tracing intertextual connections rests on the view of text as an entity that does not have an independent meaning but rather is a part of a wider textual network (Allen, 2000). In other words, texts, literary or non-literary, cannot exist as selfsufficient entities because they do not function in a closed system (Still \& Worton, 1990). This network influences the meaning of every text and discourse in an explicit or implicit way; thus, the discourse practices of a community affect the understanding of a particular text by creating systems of texts related through community practices (Lemke, 1985).

In language learning context, intertextuality is perceived as preparing learners to deal with the relations of writers' own ideas with the ideas of other authors (Blanton, 1999). This preparedness could support in various ways the learning process: (a) through incorporating reading practices that promote comprehension and writing (Ferris \& Hedgcock, 1998), (b) through engaging in writing activities that expand beyond the learners' own experiences, thus moving from the mode of writing as knowledge-telling to writing as knowledge-transformation (Bereiter \& Scardamalia, 1987), (c) through encouraging learners to engage in self-reflection (Blanton, 1999).

Intertextuality is directly connected to the literacy practices of the community. Although it is traceable in the written product (the text), it presents actually part of the writing process through revealing the engagement of the writer with other texts before, 
during, and after text creation (Blanton, 1999). Lemke (1985) proposes three specific features of texts that could help interpreting if two or more texts form a strong intertextual set: the Mode, the Tenor, and the Field. Considering these dimensions, intertextuality manifests itself "across differences of medium and genre (Mode), of role relationships and interactional ploys (Tenor), and across activity type and overt topic (Field) (Lemke, 1985, p. 279). This approach to the text would allow one to follow the text construction process, taking into account the participants: "The participants themselves, in making these texts, may make them in relation to each other, may make meanings through the relations they construe among them that penetrate into and beyond of isolated meanings of the texts by themselves" (Lemke, 1985, p. 280). Although in the above quote, Lemke does not refer specifically to language learners, it might be applicable to them based on the fact that learners create and function in discourse communities that have their specific socioeducational dimensions (Ferris \& Hedgcock, 1998; Grabe \& Kaplan, 1996).

The current study approaches intertextuality on a lexical level. This interest in lexis and its development is based on research findings that clearly show the crucial role of vocabulary in the process of the development of writing skills. Laufer (1997) summarizes SLA research that reveals lexical problems to be in the core of reading/comprehension difficulties of language learners. Haynes and Baker (1993) outlined that while factors such as syntactic complexity have impact on learners' reading comprehension, vocabulary is perceived to be the central issue. Further, based on empirical research results, researchers concluded that vocabulary accounts for the largest amount of variance in L2 students' writings (Astika, 1993; Koda 1993; Nation, 2001).

\section{The Study}

This study aims to reveal the lexical connections of the pre-writing discussion texts created in a SCMC and ACMC environments with the following drafts. Note that here the term "texts" is used in a broader sense as "records of discourse" (p. 20, Johnsotone, 2008). The researcher believes the findings of the study would help to reach the gap in the SLA research related to text development at the initial stages of the writing process and will help establishing sound practices related to the active use of CMC in the process of text construction.

The research question posed in the study is: Do the ACMC and the SCMC modes of the pre-writing discussion influence to a different degree the texts produced after those discussions on a lexical level?

\subsection{Settings and Participants}

The quantitative study presented in this paper is part of a larger mixed-design study (Sarieva, 2007). The data were collected at an urban area Intensive English Language Program (IEP) affiliated with a southeastern university in the United States. The overall 
sampling technique used to select the IEP among the numerous programs existing in the United States was purposeful sampling (Hatch, 2002; Kemper, Stringfield, \& Teddlie, 2003) which aimed to study language learning processes within contexts common for IEP settings in the United States. The program required the ESL students to be enrolled on a full-time basis receiving 25 hours of weekly instruction during a 14-week semester or to be part-time students with a minimum of 10 hours of weekly instruction during a 14week period.

The participants were 60 adult students of intermediate proficiency level as measured by Comprehensive English Language Test (CELT) and The Michigan Test of English Language Proficiency (MTELP). There were 24 female (40\%) and $36(60 \%)$ male students. The average age of the participants was 25.55 . The youngest participant was 17 years old, whereas the oldest was 46 . They came from 21 countries and spoke 13 native languages.

\subsection{Data Collection}

The study was conducted over the course of two semesters with a total of 60 participants. The data were collected during Weeks 5 and 6 of each the semester. In this stage, the students prepared for writing a paragraph by completing pre-writing activities, namely brainstorming and planning. The learners were randomly assigned into dyads and each dyad was randomly assigned to two treatment groups: an ACMC group or SCMC group. The ACMC dyads used asynchronous computer-mediated communication, while the SCMC dyads used synchronous computer-mediated communication to perform a communication task (referred henceforth as a treatment task). This task aimed at preparing the participants for writing their post-treatment paragraphs. After the task was performed, each student wrote individually the post-treatment paragraph.

\subsection{The Task Design}

The specific pre-writing task that the participants in the current study performed via CMC was a decision-making task as classified by Pica, Kanagy, and Falodun (1993). Prior to performing the task, each participant was given a set of six pictures that aimed to elicit discussion on the following topic: The People of the United States. The participants also were instructed to brainstorm together the main idea, supporting ideas, and paragraph organization of a descriptive paragraph that each one of them would write individually following the CMC discussion.

The topic was chosen considering several factors such as age appropriateness, interest, expected knowledge about US culture and history based on the curricula material covered in the writing class as well as in the other classes prior to the treatment, and on the rhetorical features of the expected post-treatment writing outcome - a descriptive paragraph. It was decided to use pictures rather than text in order to assure elicitation of student-produced language and active meaning negotiation during the discussion process.

SECTION A: theoretical papers, original research and scientific articles 


\subsection{The Study Design}

In order to answer the research question posed in the study, the pre-writing CMC interactions and the post-treatment paragraphs were examined. It is important to outline that in order to assure independence of the scores, the unit of analysis in the study was a dyad rather than an individual learner. This decision was made based on the consideration that the learners, working in dyads, influenced each other's language production.

The goal of the analysis was to find out the degree of the intertextual relationship between the CMC interactions and the post-treatment paragraphs. This analysis was based on lexical items as defined by Halliday (1989). It was considered that "all the members of a morphological paradigm are the same lexical item: for example, differ, differed, different, difference, differing, differently are all instances of one lexical item" (Halliday, 1989, p. 65). Thus, if inflectional or derivational forms with the same root were detected, they were considered to be the same lexical item. Note that the vocabulary lists used in the study, 1-999 and 1000-2000 Most Frequently Used Words Lists compiled by West and Coxhead (cited in Nation, 2001), were already sorted by lexical items.

In order to use computer software for the analysis, normalization of the spelling of the CMC scripts was necessary. The nature of this normalization was the following: the CMC interactions and the paragraphs were read by the researcher and all misspelled words were replaced with their correctly spelled counterparts while their original spelling was bracketed. For example: “... with big buildings one [nest] next to the other." After the normalization procedures were completed, the lexical items in the pre-writing discussions and the post-treatment paragraphs were identified

The content words used in the students' CMC pre-writing discussions and paragraphs that were not found in Nation's (2001) lists were identified by the researcher using a custom text analysis software designed for the study. These words were compiled into a third list named Above 2000 Words List; the words were classified by lexical items. Two independent inter-raters verified the accuracy of the Above 2000 Words List. The inter-rater reliability was higher than $99 \%$ with both inter-raters. Further, the finalized Above 2000 Words List was added to the database and was compared against the 1-999 and 1000-2000 Most Frequently Used Words Lists (Nation, 2001) aiming to assure that none of the lexical items appeared in more than one list. Thus, the researcher obtained three lexical item lists: (1) 1-999 Most Frequently Used Words Lists, compiled and published by Nation (2001), (2) 1000-2000 Most Frequently Used Words Lists, compiled and published by Nation (2001), and (3) Above 2000 Words List, compiled by the researcher. These three lists were organized by lexical items and were further used to analyze the intertextual relationship between the texts produced during the treatment (CMC interactions) and students' first post-treatment drafts.

In the analysis, each lexical item was counted only once. For example, if the words house, houses, housing appeared one or more times in either the CMC interaction or the post-treatment paragraph, they were counted as one distinct lexical item (DLI). 
Thus, the definition used for DLI was: the same lexical item that appeared one or more times in either the CMC interaction or the post-treatment paragraph present one DLI.

In order to compare the degree of the pre-writing discussion influence over the post-treatment paragraphs for the two groups participating in the study, namely the ACMC group and the SCMC group, the following steps were performed. (1) The DLIs were identified for each of the CMC pre-writing interactions and counted; this number presented the overall CMC DLI score for each dyad. (2) The DLIs were identified for each of the post-treatment paragraphs. (3) The DLIs in each post-treatment paragraph were matched against the corresponding pre-writing CMC discussion and the DLIs used in both the pre-writing CMC discussion and the post-treatment paragraphs were identified and counted obtaining a matching DLI score for each participant. (4) Further, the matching DLI scores were used to calculate the ratio of the matching DLIs to the overall CMC DLI score in the corresponding pre-writing CMC discussion. The formula used for the ratio calculation was:

$\frac{\text { Matching DLI score }}{\text { Overall CMC DLI score }}$ x $100=$ Matching DLI Ratio Score (1)

Thus, for each of the participants a matching DLI ratio score was calculated. (5) The last step was to calculate the mean matching DLI ratio score for each dyad in order to obtain independent matching DLI ratio scores that could be further used for statistical comparison. Figure 1 presents the above described steps in a flowchart format. 
Figure 1. DLI Data Analysis Flow

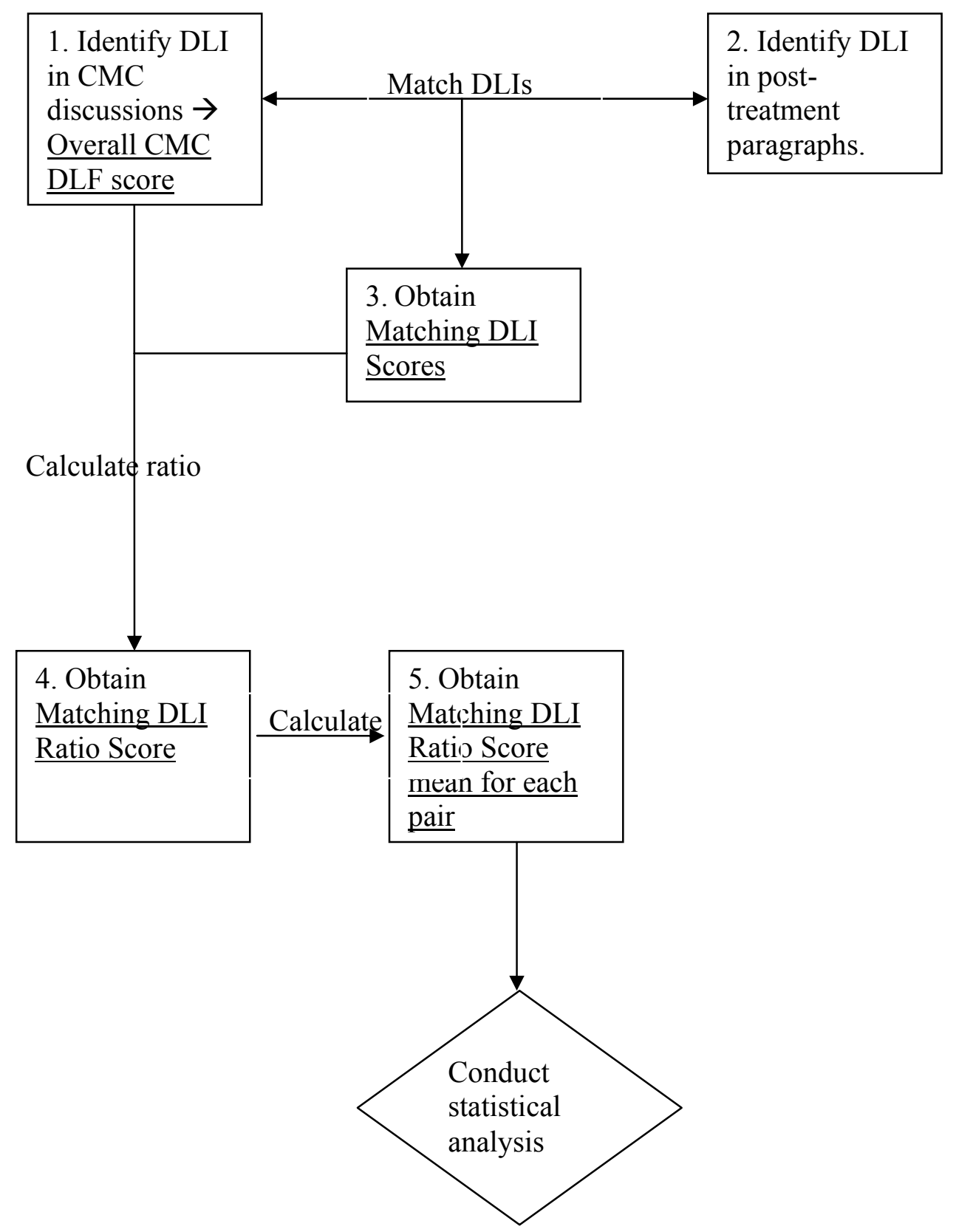

These steps were performed using the text analysis software designed for the study. In order to assure that the software scripts were running correctly, the researcher and the programmer independently calculated the scores for $10 \%$ of the data and compared them to the software outcomes. The results matched $100 \%$ which allowed the researcher to conclude that the scripts were running appropriately and all generated results were correct. 
The matching DLI ratio scores were analyzed using SAS ${ }^{\circledR}$ (SAS Institute, Inc., 2004). The ACMC and SCMC groups' means of the matching DLI ratio scores were compared through a $t$-test. The Null Hypothesis tested was that there is no significant difference between the matching DLI ratio means of the SCMC and the ACMC groups.

The goal of the test was to either confirm or reject the Null Hypothesis; in other words, to infer whether the differences between the groups' means would be "attributable to the "luck of draw" (Cody \& Smith, 2006, p. 183) or would differ in the population of English language learners of intermediate level of proficiency. The alternative to the Null Hypothesis inferred that there was a difference between the two groups' means. The researcher did not hypothesize a priori if any of the two compared means was greater than the other; thus, a two-tailed $t$-test was performed.

\subsection{The Results}

Before conducting the independent $t$-test, the researcher carefully examined the following assumptions and concluded that they were met: (1) the independence of the groups, (2) normal distribution of the sample means, and (3) equal or similar variance of the two groups. The random assignment of the participants to either ACMC or SCMC group and the consideration of the mean pair scores rather than individual scores assured the compliance with the first assumption. The comparison of the Shapiro-Wilk W=0.6772 for the ACMC group and Shapiro-Wilk W=0.7125 for the SCMC group with the SAS default level of significance, alpha $=.05$, allowed to conclude with $95 \%$ confidence that there was a sufficient evidence that the means were normally distributed. Finally, the probability $F$ value of 0.1128 was obtained; this $F$ value was greater than the SAS default level of significance, alpha $=.05$, which allowed to conclude that equal group variances and probability values must be used when interpreting the test outcomes.

For the matching DLI ratio scores, a two-tailed $t$-test was conducted to compare the means of the ACMC group $(M=40.42, S D=13.66)$ and SCMC group $(M=26.30$, $S D=8.63)$. The Null Hypothesis was rejected at $\alpha=.05$ level of significance, $t(28)=$ $3.25, p=.003$. The Mean difference was 14.11 at $95 \%$ confidence interval $(5.23,22.99)$. The Cohen's $d(1.20)$ signified a large effect size.

Based on these results, the researcher concluded, that the probability of obtaining higher matching DLI ratio score means for the ACMC group than for the SCMC group from a representative population under the same conditions was highly unlikely. Thus, the intertextual connections as measured with matching DLI ratio scores between the prewriting interactions and the first drafts for the ACMC group participants were significantly stronger than for their SCMC counterparts. The descriptive statistics are presented in Table 1 and the outcomes of the $t$-test are presented in Table 2. 
Table 1

Independent t-test of the Distinct Lexical Items (DLIs): Descriptive Statistics

\begin{tabular}{cccccc} 
& N & DLI M & DLI SD & DLI Skewness & DLI Kurtosis \\
& & & & & \\
\hline ACMC & 17 & 40.42 & 13.66 & 0.02 & -0.93 \\
SCMC & 13 & 26.30 & 8.63 & 0.42 & -0.42
\end{tabular}

Table 2

Independent t-test of the Distinct Lexical Items (DLI): Statistics and Outcomes

\begin{tabular}{ccccc} 
DLI & DF & $\begin{array}{c}\text { Null Hypothesis: } \mathrm{H}_{0}: \mu_{1}-\mu_{2}=0, \\
\alpha=.05\end{array}$ & $\begin{array}{c}\text { Probability } P, \\
\alpha=.05\end{array}$ & Variance \\
\hline $\begin{array}{c}\text { ACMC } \\
\text { SCMC }\end{array}$ & 28 & Rejected $(\mathrm{p}=.0030)$ & 0.1128 & Equal
\end{tabular}

\section{Summary of the Findings and Discussion}

The results of the DLI scores $t$-test suggested that, as measured by DLIs, the ACMC and the SCMC modes of the pre-writing discussion influenced to a different degree the texts produced after these discussions. In the post-treatment writings, the students who participated in the ACMC pre-writing discussions used to a greater extent the DLIs from their pre-writing discussions as compared to the SCMC group. Thus, it was concluded that the intertextual relation on a lexical level between the pre-writing discussion performed via ACMC mode (treatment) and the writings that followed these discussions (post-treatment) was stronger as compared to the same criteria for the SCMC group.

This comparison shed some light on the intertextual relationships between these texts. It allowed inferring that the degree to which the CMC pre-writing discussion influenced the first draft on a lexical level was affected by the mode of the pre-writing discussion; more specifically, the ACMC pre-writing discussion seemed to influence to a higher degree the first drafts. This conclusion is based on the assumption that the same DLIs might have conveyed similar or identical ideas given that these were "content words" (Holiday, 1989, p. 63) used within the same context of the assigned topic.

These results could be interpreted in the light of the qualitative findings about the differences in ACMC and SCMC pre-writing interactions reported by Sarieva (2007). The extent to which the pre-writing interactions influenced students' first drafts seem to be grounded in the specifics of the language produced during the pre-writing discussion in both CMC environments. According to Sarieva (2007), the ACMC interactions were task- rather than interaction-oriented; they resembled more advanced steps of the writing process such as outlining and first draft production. This resemblance could have made it easier for the participants to incorporate the vocabulary of their ACMC discussions into 
their first drafts. Further, taking into account the high interactivity of the SCMC postings (Smith, 2003), it could be inferred it influenced negatively the intertextual connections on a lexical level. These connections could have been restricted by the production of fewer vocabulary items that were inherent to the task and thus could be readily used in the first drafts.

\section{Pedagogical Implications}

Although pre-writing and drafting in the context of the writing process have not been addressed specifically in the SLA writing research or in ESL writing text books, these stages are presented in the literature (Ferris \& Hedgcock, 1998; Grabe \& Kaplan, 1996; Smith, 1996) and activities to be completed are suggested in the second language writing textbooks. The outcomes of the current research provide some insights on the intertextual connections existing between $\mathrm{CMC}$ pre-writing discussions and subsequent drafting. These outcomes could further inform the pedagogical practices of writing teachers especially when the instruction is delivered in a web enhanced or distance learning environments. However, it should be noted that the use of computer mediated discussions has its place in face-to-face classrooms as well.

CMC activities can support the collaborative planning and idea generation during the pre-writing stage even when the collaborators are separated by time and space. However, the instructor should carefully consider the mode of the CMC interaction in the design of the pre-writing task. The interactive nature of the SCMC discussion might be more appropriate when ideas related to the future writing project are generated, while later, when these ideas need to be refined, the ACMC mode of peer collaboration should be considered.

\section{Implications for Further Research}

The understanding of writing as a process rather than a product has reshaped the way researchers and practitioners in the field of SLA approach writing (Grabe \& Kaplan,1996; Hyland \& Hyland, 2006a). In addition, the recognition of the strong influences of social environments on this process offered new venues for research that informed the pedagogical practices in the second language classroom. Peer and student/teacher interactions remain in the focus of writing research for more than a decade now (Ferris, 2003; Grabe \& Kaplan, 1996; Hyland \& Hyland, 2006a,b). However, most of the research has been focused on revising and editing which are the later stages of the writing process. In the field of SLA, there is a limited body of research on the initial part of the writing continuum: the pre-writing and drafting. Thus, more research, both quantitative and qualitative, that would address the specifics, dynamics, and relations of pre-writing and drafting is warranted.

Multiple questions await to be addressed in future research. How do students progress through these first stages of the process? What is the role of peer collaboration 
and of teacher involvement during the pre-writing and drafting stages? How do students perceive and participate in peer interactions during the pre-writing stage in face-to-face and CMC environments? What is the teacher's role in these early stages of the process? How do the students benefit from different pre-writing tasks and in different learning environments? Are pre-writing tasks such as individual and group brainstorming, mapping, and outlining interchangeable or could they support only a specific sub-stage of pre-writing? How could language acquisition in terms of vocabulary and specific grammar forms be supported in the pre-writing processes and further transferred to drafting? How could writing skills development in terms of text organization, rhetorical soundness, and reader consideration be supported at these stages of the writing process?

In addition, the active inclusion of new technologies into the language learning process poses additional issues for future research in the context of the writing process. The role of computer mediated interactions and the specifics of these interactions in different modes when initiating the writing process could be addressed. Accounting for the text-based nature of the computer mediated communication, this study addressed the intertextual connections between the pre-writing discussions and the first drafts of language learners; in future research, it could be instrumental to attend in more detail to the degree and the specifics of language transfer that takes place in the beginning of the writing process continuum.

\section{References}

Allen, G. (2000). Intertextuality. Routledge.

Astika, G. G. (1993). Analytical assessment of foreign students' writing. RELC Journal, 24, 61-72.

Bereiter, C., \& Scardamalia, M. (1987). The psychology of written composition. Hillsdale, NJ: L. Erlbaum.

Chapelle, C. A. (2000). Is network-based learning CALL? In M. Warschauer \& R. Kern (Eds.) Networkbased language teaching: Concepts and Practice, (pp. 204-228). Cambridge: Cambridge University Press.

Cody, R. P., \& Smith, J. K. (2006). Applied statistics and the SAS programming language (5th ed.). Upper Saddle River, NJ: Prentice Hall.

Faigley, L. (1994). Competing theories of process: A critique and a proposal. In S. Perl (Ed.), Landmark essays on writing process (pp. 149-164). Hermagoras Press.

Ferris, D. (2003). Response to student writing: Implications for second-language students. Mahwah, NJ: Lawrence Erlbaum Associates.

Ferris D., \& Hedgcock J. S. (1998). Teaching ESL composition. Mahwah, NJ: Lawrence Erlbaum Associates.

Grabe W., \& Kaplan R. B. (1996). Theory and practice of writing. London: Longman.

Halliday, M. A. K. (1989). Spoken and written language. Oxford: Oxford University Press.

Haynes M., \& Baker, I. (1993). American and Chinese readers learning from lexical familiarization in English texts. In T. Huckin, M. Haynes, \& J. Coady (Eds.), Second language reading and vocabulary acquisition (pp. 130-152). Norwood: Ablex.

Hyland, K. \& Hyland, F. (2006a). Feedback in second language writing: Contexts and issues. Cambridge: Cambridge University Press.

Hyland, K. \& Hyland, F. (2006b). Contexts and issues in feedback on L2 writing: An introduction. In K. Hyland \& F. Hyland (Eds.), Feedback in second language writing: Contexts and issues (pp. 115). Cambridge: Cambridge University Press.

SECTION A: theoretical papers, original research and scientific articles 
Jepson, K. (2005). Conversations and negotiated interactions in text and voice chat rooms. Language learning and technology, 9(3), 79-98.

Jin, Li. (2007).Computer-mediated peer response in an ESL academic writing class: cultural historical activity theoretical perspective. Dissertation, University of South Florida, Tampa.

Johnstone, B. (2008). Discourse Analysis (2nd ed.). Blackwell Publishing.

Kasper, L. F. (2000). New technologies, new literacies: Focus discipline research and ESL learning communities. Language learning and technology, 4(2), 105-128. Retrieved on January, 04 from: http://1t.msu.edu/vol4num2/kasper/default.html.

Kent, T. (1999). Introduction. In T. Kent (Ed.), Post-Process Theory (pp. 1-6). Southern Illinois University Press.

Kern, R. (1995). Restructuring classroom interaction with network computers: effects on quantity and characteristics of language production. Modern Language Journal, 79, 457-476.

Koda, K. (1993). Task-induced vocabulary in FL composition: Language specific perspectives. Foreign language annals, 26, 332-346.

Laufer, B. (1997). The lexical plight in second language reading: Words you don't know, words you think you know, and words you can't guess. In J. Coady \& T. Huckin (Eds.), Second language vocabulary acquisition: A rationale for pedagogy (pp. 20-34). United Kingdom: Cambridge University Press.

Lemke, L. J. (1985). Ideology, intertextuality, and the notion of register. In J. D. Benson \& S. G. Greaves (Series Eds.), Advances in Discourse processes series: Vol. 15 Systemic perspectives on discourse: Vol. 1 (275-294). Ablex Publishing.

Leu, J., \& Donald J. (2000). Literacy and technology: Deictic consequences for literacy education in an information age. In M. L. Kamil, P. Mosenthal, P. D. Pearson \& R. Barr (Eds.), Handbook of reading research, Volume III. Mahway, NY: Lawrence Erlbaum Associates.

Matsuda, P. K. (2003). Process and post-process: A discursive history. Journal of Second Language Writing, 12(1), 65-83.

Miller, L. (2007). Review of calling on CALL: From theory and research to new directions in foreign language teaching. Language Learning and Technology, 11(1), 40-44.

Nation, I. S. P. (2001). Learning vocabulary in another language. Cambridge: Applied Linguistics.

Petraglia, J. (1999). Is there life after process? The role of social scientism in changing discipline. In T. Kent (Ed.), Post-Process Theory (pp. 49-64). Southern Illinois University Press.

Pica, T., Kanagy, R., \& Falodun, J. (1993). Choosing and using communication tasks for second language instruction and research. In G. Crookes \& S. M. Gass (Eds.), Tasks and language learning: Integrating theory and practice (pp. 9-34). Clevedon, UK: Multilingual Matters.

Reither, J. A. (1994). Writing and knowing: Towards redefining the writing process. In S. Perl (Ed.), Landmark essays on writing process (pp. 141-148). Hermagoras Press.

Sarieva, I. (2007). The Communicative Two-Way Pre-Writing Task Performed via Asynchronous and Synchronous Computer-Mediated Communication and its Influence on the Writing Expertise Development of Adult English Language Learners: A Mixed Design Study. D University of South Florida, Tampa.

SAS Institute Inc. (2004). SAS ${ }^{\circledR}$ (Version 9.1.3) [Computer software]. Language Reference: Concepts. Cary, NC: SAS Institute Inc.

Silva, T., \& Matsuda, P. K. (Eds.). (2001). On second language writing. Mahwah, NJ: Lawrence Erlbaum Associates.

Sotillo, M. S. (2000). Discourse functions and syntactic complexity in synchronous and asynchronous communication. Language Learning and Technology 4(1), 82-119.

Still, J., \& Worton, M. (1990). Introduction. In M. Worton \& J. Still (Eds.), Intertextuality: Theories and practices (pp. 1-44). Manchester University Press. 
Swaffar, J. (1998). Networking language learning: Introduction. In J. Swaffar, S. Romano, P. Markley, \& $\mathrm{K}$. Arens (Eds.), Language learning online: Theory and practice in the ESL and L2 computer classroom (pp. 1-15). Austin, TX: Labyrinth.

Warschauer, M. (1999). Electronic literacies: Language, culture, and power in online education. Mahwah, NJ, London: Lawrence Erlbaum Associates, Publishers.

Warschauer, M. (2004). Technology and writing. In C. Davidson and J. Cummins (Eds.), Handbook of English language teaching. Kluwer: Dordrecht, Netherlands. Retrieved on November 15, 2004, from: http://www.gse.uci.edu/markw/technology.pdf.

Warschauer, M. (2006). Literacy and technology: Bridging the divide. In D. Gibbs \& K.-L. Krause (Eds.), Cyberlines 2: Languages and cultures of the Internet, (pp. 163-176). Albert Park, Australia: James Nicholas. 